\title{
Assessment of rapid methods of pneumococcal antigen detection in routine sputum bacteriology
}

\author{
MICHAEL WHITBY, KARL G KRISTINSSON, MORAG BROWN \\ From the University Department of Bacteriology, Royal Infirmary, Glasgow G4 OSF
}

SUMMARY Sputum specimens from 480 patients were examined for the presence of pneumococci by Gram film and culture and for pneumococcal antigen by counterimmunoelectrophoresis, coagglutination, and latex agglutination. Ninety six positive specimens were detected. Gram film and culture provided the most reliable techniques in well taken specimens collected early in the illness before antibiotic treatment had started. More than $70 \%$ of the specimens examined were submitted after starting antibiotics, however, and in these specimens, methods of antigen detection proved of greater value than either Gram film or culture. Counterimmunoelectrophoresis, coagglutination, and latex agglutination were similar in sensitivity and specificity, but coagglutination and latex agglutination were much easier to perform and to read.

The rapid diagnosis of pneumococcal chest infection by examination of expectorated sputum is valuable for the early institution of appropriate antimicrobial treatment. Gram stained smears are a traditional method of providing a rapid diagnosis, but increasingly clinical laboratories are applying techniques of antigen detection to routine sputum samples. Such methods include counterimmunoelectrophoresis, coagglutination, and latex agglutination, the last two now being available in commercial kits. At present, however, no manufacturer specifically recommends his product for the rapid identification of pneumococcal antigen in sputum or other respiratory secretions.

Where sputum is obtained from a patient already taking antibiotics counterimmunoelectrophoresis is a reliable and sensitive method of demonstrating pneumococcal antigen. ${ }^{2}$ Coagglutination appears to be faster and simpler, and is believed to be both more specific and more sensitive than counterimmunoelectrophoresis. ${ }^{3}$ There are no published assessments of the value of latex agglutination in sputum bacteriology, but this technique has been favourably compared with coagglutination in detection of pneumococcal antigen in cerebrospinal fluid, serum, and urine. ${ }^{4}$

We have compared the three methods of pneumococcal antigen detection in unselected sputum specimens sent to a hospital laboratory for

Accepted for publication 6 November 1984 routine bacteriological culture and have assessed the findings in relation to those obtained by microscopy and culture.

\section{Material and methods}

\section{SPUTUM SPECIMENS}

One thousand and ninety seven samples randomly selected from all sputum samples sent to our laboratory over 4 months were assessed for purulence and degree of oropharyngeal contamination according to the method of Bartlett et al. ${ }^{5}$ Four hundred and eighty specimens were considered suitable for further processing in that they contained mucopurulent material and had at least 10 leucocytes/low power field and fewer than 25 epithelial cells/low power field. All specimens were further examined microscopically by Ziehl-Neilsen staining to exclude the presence of acid fast bacilli, before being homogenised with an equal volume of Sputolysin (Calbiochem-Behring Corporation, La Jolla, California) and allowed to equilibrate at $37^{\circ} \mathrm{C}$ for $30 \mathrm{~min}$. Non-quantitative culture for pneumococci was undertaken on Columbia agar plates containing 5\% horse blood (Gibco Europe, Paisley), which were incubated at $37^{\circ} \mathrm{C}$ in air, in 5-10\% $\mathrm{CO}_{2}$, and anaerobically for 18-24 h. Pneumococci were identified by colony morphology and optochin sensitivity. After culture all specimens were immediately frozen at $-20^{\circ} \mathrm{C}$ until further processing was undertaken. 


\section{ANTIGEN DETECTION}

The frozen specimens were allowed to thaw and were then heated to $80^{\circ} \mathrm{C}$ for at least $10 \mathrm{~min}$ to try to reduce false positive reactions due to non-specific precipitation. The counterimmunoelectrophoresis, coagglutination, and latex agglutination tests were carried out independently of each other; the examiners did not know the origin of the specimen nor the results of other tests.

\section{Counterimmunoelectrophoresis}

The method used was that of El Refaie and Dulake, with each well containing $5 \mu$ l of homogenised sputum or $5 \mu$ l of antiserum (Omniserum, State Serum Institute, Copenhagen, Denmark). Each plate was read immediately and then held at $4^{\circ} \mathrm{C}$ before examining with $\mathrm{a} \times 10$ hand lens at $4 \mathrm{~h}$ and 24 h. All samples found positive by the above method which were not positive by any other techniques were rerun against the immunoglobulin fraction of normal rabbit serum obtained from DAKO (Mercia Brocades Ltd, Surrey). Samples positive with rabbit immunoglobulin were regarded as negative reactions.

\section{Coagglutination}

The Phadebact Pneumococcus test (Pharmacia Diagnostics AB, Uppsala, Sweden) was used. The coagglutination reagent is prepared by sensitising heat killed Staphylococcus aureus Cowan 1 strain with Omniserum. All specimens were treated with both this reagent and a negative control included in the kit. The negative control is prepared by coating Cowan I staphylococci with gammaglobulin from non-immunised rabbits. All samples for which results of coagglutination differed from those of counterimmunoelectrophoresis or latex agglutination or both were ultimately re-examined by all three methods.

\section{Latex agglutination}

A Wellcogen Streptococcus pneumoniae kit (Wellcome Diagnostics, Beckenham, UK) was used; the agglutination reagent is prepared by sensitising a suspension of polystyrene latex particles with Omniserum. All sputum samples were tested both with this reagent and with a negative control prepared by coating latex particles with gammaglobulin from non-immunised rabbits. Samples in which results differed from counterimmunoelectrophoresis or coagglutination or both were ultimately reexamined by all three methods.

The specificity and sensitivity of each test was calculated. Sensitivity is defined as "the percent of positive results in patients with the disease" and specificity as "the percent of negative results among people who do not have the disease"?

The $\chi^{2}$ test or where appropriate Fisher's exact probability test (with Tochers modification) was used for statistical analysis.

\section{CLINICAL CORRELATION}

A specimen was considered to be positive when either of two criteria were fulfilled: $(a)$ positive culture or (b) any two other tests positive.

A random two in three selection of case notes was made of all patients with positive sputum samples. Clinical history was reviewed to determine: (a) probable diagnosis of respiratory infection and $(b)$ prescription of antibiotics before collection of the sputum specimen.

\section{Results}

Of the 480 specimens tested, 96 from 96 patients fulfilled the criteria of a positive sample. Two were positive by culture only, in 34 pneumococcus or antigen was detected by only two methods, and the remaining 60 specimens were positive by three or more techniques. Gram film alone was positive in two cases, coagglutination alone in nine, latex agglutination only in four, and all of these reactions together with seven specimens positive in counterimmunoelectrophoresis against Omniserum only were regarded as false positive results. Ten specimens were positive against Omniserum and rabbit immunoglobulin and these, together with sputa in which autoagglutination occurred (11 with coagglutination and eight with latex agglutination) were treated as negative. The Figure shows the detailed inter-relation of all true positive results.

The sensitivity and specificity of each method are shown in Table 1. Differences between the three techniques of antigen detection were not statistically significant $(p>0 \cdot 05)$.

Review of 66 case notes from the 96 patients with positive sputum samples showed clinical diagnoses of lobar or bronchopneumonia (17), acute or chronic bronchitis (17), postoperative chest infection (24), and unspecified chest infection (8). Seventy per cent of sputum specimens received were submitted after antibiotics had been started. Each method of pneumococcal detection was correlated with the presence or absence of previous antimicrobial treatment (Table 2).

In patients in whom sputum was collected before antibiotics were started (mainly those with pneumonia or acute bronchitis) we were unable to show significant differences between Gram film and culture or between counterimmunoelectrophoresis, coagglutination, and latex agglutination. Antigen 
Inter-relation of positive results

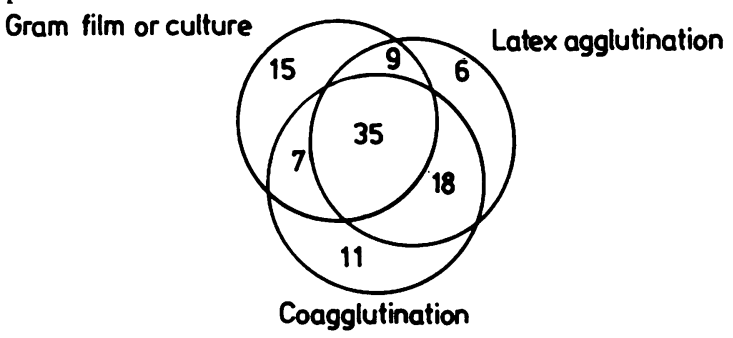

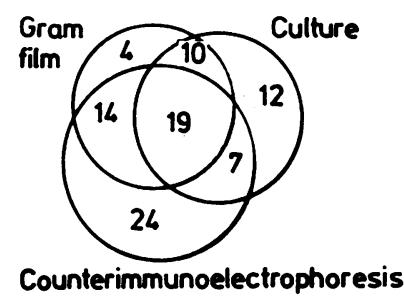

detection techniques, however, were considerably less reliable than either Gram film or culture in these cases.

Table 1 Overall sensitivity and specificity of techniques used

\begin{tabular}{llc}
\hline Detection method & $\begin{array}{l}\text { Sensitivity } \\
(\%)\end{array}$ & $\begin{array}{l}\text { Specificity } \\
(\%)\end{array}$ \\
\hline Gram film & 49 & 99 \\
Culture & 50 & 100 \\
Counterimmunoelectrophoresis & 67 & 98 \\
Coagglutination & 74 & 98 \\
Latex agglutination & 71 & 99
\end{tabular}

Results expressed as a \% of 96 sputum specimens which were positive by definition.

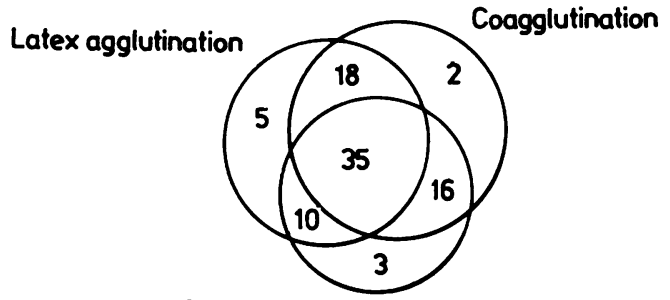

Counterimmuno electrophoresis

Table 2 Detection of pneumococcus or pneumococcal antigen and relation to antibiotic treatment

In patients whose sputum was taken after the start of antibiotic treatment (mainly those with postoperative chest infection or chronic bronchitis) Gram film was significantly better than culture. Differences between counterimmunoelectrophoresis, coagglutination, and latex agglutination were not shown, but each of these three methods was significantly more sensitive than either Gram film or culture.

Combination of each antigen detection technique with results for Gram film, either in the total series or in its subgroups, failed to distinguish a significantly more reliable method.

Sputum samples collected before antibiotic treatment $(n=20)$

\begin{tabular}{llll}
\hline Detection method & No positive & $\%$ & Statistical significance compared with culture \\
\hline Gram film & 15 & 75 & NS \\
Culture & 17 & 85 & - \\
Counterimmunoelectrophoresis & 11 & 55 & $\mathrm{p}<0.01$ \\
Coagglutination & 13 & 65 & $\mathrm{p}<0.01$ \\
Latex agglutination & 12 & 60 & $\mathrm{p}=0.02$ \\
\hline
\end{tabular}

Sputum samples collected after antibiotic treatment $(n=46)$

\begin{tabular}{|c|c|c|c|}
\hline & & & $\begin{array}{l}\text { Statistical significance compared with } \\
\text { counterimmunoelectrophoresis }\end{array}$ \\
\hline $\begin{array}{l}\text { Gram film } \\
\text { Culture } \\
\text { Counterimmunoelectrophoresis } \\
\text { Coagglutination } \\
\text { Latex agglutination }\end{array}$ & $\begin{array}{r}15 \\
8 \\
29 \\
31 \\
31\end{array}$ & $\begin{array}{l}33 \\
17 \\
63 \\
67 \\
67\end{array}$ & $\begin{array}{l}\mathrm{p}<0.001 \\
\mathrm{p}<0.001 \\
\overline{N S} \\
\mathrm{NS}\end{array}$ \\
\hline
\end{tabular}

NS $=$ not significant. 


\section{Discussion}

Our study has shown that counterimmunoelectrophoresis, coagglutination, and latex agglutination are similarly sensitive and specific for the detection of pneumococcal antigen in expectorated sputum. A major difficulty in comparing these antigen detection techniques lies in the definition of a true positive result. This has been overcome in previous studies by assessing only patients with radiological abnormalities or patients with positive blood cultures. Radiographic changes may not be present in early pneumococcal chest infection, and only 30$40 \%$ of patients have positive blood cultures. These groups may represent special subpopulations. ${ }^{8}$ We believe that our definition of a positive result provides a reliable assessment of specificity and sensitivity. This definition may have excluded genuine positive results; however, inclusion of specimens in which only one non-culture technique was positive in our assessment did not significantly alter our conclusions.

We are unable to confirm the results of Kalin et $\mathrm{al}^{3}$ that coagglutination is a more sensitive technique in sputum investigation than counterimmunoelectrophoresis. Furthermore, we may have underestimated both the sensitivity and specificity of counterimmunoelectrophoresis as our method is known to have a low detection rate of negatively charged pneumococci, particularly serotypes 7 and 14. ${ }^{9}$ Our assessment of counterimmunoelectrophoresis must be regarded as conservative. Comparison of these three antigen detection techniques is to some extent a comparison of Omniserum antiserum. Both commercial manufacturers as well as our own counterimmunoelectrophoresis method use this product, which is recognised to vary slightly from batch to batch and will therefore influence reported sensitivity and specificity in any study.

Surprisingly, over $70 \%$ of specimens sent to our laboratory were from patients taking antibiotics, and in these specimens culture and Gram film yielded poor results. This is a finding common to other studies $^{310}$ and emphasises the need for a reliable antigen detection method in such specimens. We analysed only a small series of positive samples submitted before antibiotic treatment, but even so our conclusions agree with those of other studies ${ }^{11}$ that Gram film and culture are reliable techniques in these cases but antigen detection methods show low sensitivity. This poor antigen detection rate may occur because specimens are submitted at an early stage of illness when antigen concentration in sputum has not reached detectable levels.

In general, both coagglutination and latex agglutination results were easier to read than counterimmunoelectrophoresis results, with a positive reaction appearing within $90 \mathrm{~s}$ and being clearly visible to the naked eye. The number of specimens in which autoagglutination prevented a reliable result was less than $0.5 \%$ from both coagglutination and latex agglutination provided the specimen was pretreated by heating.

Our study confirms that Gram film (and culture) are reliable methods in well collected specimens taken early in the illness before antibiotic treatment. Antigen detection methods do not appreciably increase yield in this situation. In sputum samples from patients already taking antibiotics pneumococcus is best detected with antigen screening techniques. To apply these methods routinely is both expensive and time consuming and further assessment of the value of such results to the clinician is needed. Counterimmunoelectrophoresis appears to have significant advantages where there is a large number of specimens. In individual cases where a rapid result is necessary to resolve a therapeutic or diagnostic dilemma, however, both coagglutination and latex agglutination provide easily read and accurate results.

\section{References}

' Dulake C. Countercurrentimmunoelectrophoresis for the diagnosis of pneumococcal chest infection. J Infect 1979; 1 (suppl 2):45-51.

${ }^{2}$ Kaijser B, Berntsson E, Trollfors B. Serological diagnosis of pneumococcal pneumonia. J Infect 1979; 1 (suppl 2):39-44.

${ }^{3} \mathrm{Kalin} \mathrm{M}$, Lindberg AA, Olausson EH. Diagnosis of pneumococcal pneumonia by coagglutination and counterimmunoelectrophoresis of sputum samples. Eur J Clin Microbiol 1982; 1:91-6.

4 Ingram DL, Pearson AW, Occhiuti AR. Detection of bacterial antigens in body fluids with the Wellcogen Haemophilus infuenzae $b$, Streptococcus pneumoniae, and Neisseria meningitidis (ACYW 135) latex agglutination tests. J Clin Microbiol 1983;18:1119-21.

${ }^{5}$ Bartlett RC. Medical microbiology: quality, cost and clinical relevance. New York: John Wiley \& Sons, 1974:24-31.

- El-Refaie M, Dulake C. Counter-current immunoelectrophoresis for the diagnosis of pneumococcal chest infection. $J$ Clin Pathol 1975; 28:801-6.

${ }^{7}$ Kreig AF, Cambing R, Falen RS. Why are clinical laboratory tests performed? When are they valid? JAMA 1975;233:76-8.

${ }^{8}$ Austrian R, Gold I. Pneumococcal bacteremia with especial reference to bacteremic pneumococcal pneumonia. Ann Int Med 1964;60: 759-76.

9 Anhalt JP, Yu PKW. Counterimmunoelectrophoresis of pneumococcal antigens: improved sensitivity for detection of types VII and XIV.J Clin Microbiol 1975;28:510-5.

${ }^{10}$ Spencer RC, Philp JR. Effect of previous antimicrobial therapy on bacteriological findings in patients with primary pneumonia. Lancet 1973;ii:349-51.

" Halin M, Lindberg AA. Diagnosis of pneumococcal pneumonia: A comparison between microscopic examination of expectorate, antigen detection and cultural procedures. Scand J Infect Dis 1983; 15:247-55.

Requests for reprints to: Dr M Whitby, Department of Microbial Diseases, City Hospital, Hucknall Road, Nottingham NG5 1PB, England. 